Miguel Almiron, Esther Jacopin, Giusy Pisano (dir.), Stéréoscopie et illusion. Archéologie et pratiques contemporaines: photographie, cinéma, arts numériques Villeneuve-d'Ascq, Presses universitaires du Septentrion, 2018

\title{
Marie Auger
}

\section{(2) OpenEdition}

Édition électronique

URL : http://journals.openedition.org/artefact/5207

DOI : 10.4000/artefact. 5207

ISSN : 2606-9245

Éditeur :

Association Artefact. Techniques histoire et sciences humaines, Presses universitaires du Midi

Édition imprimée

Date de publication : 15 décembre 2019

Pagination : 386-388

ISBN : 978-2-8107-0667-9

ISSN : 2273-0753

Référence électronique

Marie Auger, « Miguel Almiron, Esther Jacopin, Giusy Pisano (dir.), Stéréoscopie et illusion. Archéologie et pratiques contemporaines : photographie, cinéma, arts numériques », Artefact [En ligne], 11 | 2019, mis en ligne le 27 novembre 2020, consulté le 30 novembre 2020. URL : http://journals.openedition.org/ artefact/5207; DOI : https://doi.org/10.4000/artefact.5207

Ce document a été généré automatiquement le 30 novembre 2020.

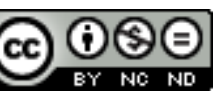

Artefact, Techniques, histoire et sciences humaines est mise à disposition selon les termes de la Licence Creative Commons Attribution - Pas d'Utilisation Commerciale - Pas de Modification 4.0 International. 


\title{
Miguel Almiron, Esther Jacopin, Giusy Pisano (dir.), Stéréoscopie et illusion. Archéologie et pratiques contemporaines : photographie, cinéma, arts numériques
}

Villeneuve-d'Ascq, Presses universitaires du Septentrion, 2018

\author{
Marie Auger
}

\section{RÉFÉRENCE}

Miguel Almiron, Esther Jacopin, Giusy Pisano (dir.), Stéréoscopie et illusion. Archéologie et pratiques contemporaines : photographie, cinéma, arts numériques, Villeneuve-d'Ascq, Presses universitaires du Septentrion, 2018, 266 p.

1 En 2015, le projet «Les Arts Trompeurs. Machines, Magie, Médias » recevait le soutien du Labex Arts-H2H pour une durée de trois ans. Porté par Jean-Marc Larrue et Giusy Pisano, il avait pour objectif d'analyser un certain nombre de techniques mises au service des arts du spectacle, en Europe et en Amérique du Nord, sous l'angle de la magie. Une série d'événements scientifiques ont eu lieu dans le cadre de ce programme de recherche dont le colloque "Stéréoscopie et illusion ». Organisé en 2016 à la Fémis et à l'ENS Louis-Lumière par Miguel Almiron, Esther Jacopin, Giusy Pisano, il fournit l'essentiel de la matière à Stéréoscopie et illusion. Archéologie et pratiques contemporaines: photographie, cinéma, arts numériques, paru deux ans plus tard sous leur direction.

L'ouvrage comporte trois contributions supplémentaires comparativement au colloque et quatre parties au lieu de six. La première partie est consacrée à la stéréoscopie du point de vue de la perception. Elle permet de rentrer dans le sujet par un biais physiologique et donc incarné. La deuxième et la troisième parties abordent la 
stéréoscopie appliquée aux deux médias qui l'ont popularisée : la photographie et le cinéma. Et pour finir, la quatrième ouvre sur l'actualité de la réalité virtuelle et du son binaural dans le contexte des arts numériques. La progression est cohérente et permet d'aborder la stéréoscopie dans deux acceptions - vision binoculaire ou technique au service d'une illusion spatiale.

3 Comme le colloque avant lui, la publication Stéréoscopie et illusion ouvre un espace de réflexion interdisciplinaire, intergénérationnel, international et paritaire. Il comprend des contributions d'artistes, de praticiens ainsi que de chercheurs affiliés à une grande variété d'institutions. Les universités parisiennes Évry Val d'Essonne, Paris-Est Marnela-Vallée, Paris 8 et Paris 3 sont représentées aux côtés des universités Lyon 2 et Lille 3 , de la Queen Mary Université de Londres, de l'Universidade NOVA de Lisbonne, de l'Université Laval de Québec, de l'Université du Connecticut, de l'Université Libre de Bruxelles et de l'École du Louvre. Par ailleurs, les femmes et les hommes sont représentés à parts égales et de jeunes doctorants ont été invités pour faire leurs armes. La recherche d'équilibre se manifeste à tous points de vue.

La partie de l'ouvrage qui a le plus bénéficié de cette mixité est celle dédiée au cinéma. Les textes qui la composent se renforcent mutuellement dans leurs analyses et le lecteur peut ainsi garder quelques idées maitresses à l'esprit. La plus percutante concerne la nature même de l'illusion procurée par la vision stéréoscopique. Celle-ci ne vise pas à se rapprocher au plus près de la vision humaine (thèse naturalise la plus répandue), mais à procurer des sensations spatiales inédites. Elle est une «illusion partielle » explique l'universitaire Nick Jones et cette partialité n'est pas le signe de son insuffisance mais au contraire, la condition même d'un écart expressif. À cette idée forte, s'ajoute une série de considérations captivantes à propos de l'économie, de la technique et de l'esthétique du cinéma en 3D. Le lecteur se voit expliquer de façon didactique qu'un film peut être réalisé en 3D «native » ou bien converti en 3D, après coup, afin de réaliser des bénéfices commerciaux. Il lui est également donné à comprendre les évolutions non linéaires du cinéma stéréoscopique sur les marchés internationaux depuis le succès d'Avatar (2009) et le développement des techniques numériques. Mais ce n'est pas tout, grâce à l'analyse de quelques films d'auteur comme Pina de Wim Wenders, il prend connaissance des efforts déployés par certains réalisateurs pour explorer les potentialités expressives propres à ce médium. S'il n'en avait pas déjà conscience, il lui apparaît donc clairement que le cinéma en 3D ne se limite pas à quelques effets de jaillissements.

Deux tables rondes du colloque ont été également retranscrites dans cette partie. Il s'agit d'un échange avec le réalisateur Jean-Pierre Jeunet et cinq stéréographes. Leurs témoignages occasionnent quelques redondances avec les essais universitaires mais ils présentent plusieurs intérêts. Sous forme de dialogues vivants, ils rythment intelligemment l'ouvrage. Parce qu'ils fourmillent de détails inattendus propres aux gens de métier, ils donnent l'impression d'être dans un making-of. Divertissants, ils offrent par ailleurs une possibilité supplémentaire de devenir un spectateur averti. Malheureusement, les qualités de cette partie dédiée à la stéréoscopie au cinéma ne caractérisent pas l'ensemble de la publication. Après l'introduction générale signée Almiron, Jacopin et Pisano, le lecteur bute sur la technicité de la première contribution. Censée l'introduire aux enjeux physiologiques de la vision stéréoscopique, elle multiplie les graphiques mais manque cruellement de didacticité. À ce problème de vulgarisation, s'ajoutent parfois ceux de méthode. La partie consacrée à la stéréoscopie 
en photographie compile des textes de factures inégales. Seul l'article de Kim Timby allie l'élégance du raisonnement à l'originalité. En prenant appui sur des vues réalisées au Quinétoscope dans les années 1850 et des autostéréoscopies réalisées entre les années 1900 et 1940, l'universitaire démontre efficacement qu'il n'y a pas eu « une, mais des illusions stéréoscopiques à travers l'histoire de la photographie ».

Pour finir, notons que la partie consacrée aux arts numériques supporte mal le format de l'édition papier. Les trois textes qui la composent présentent chacun une création contemporaine : une interaction gestuelle entre un humain et un acteur virtuel réalisée dans le cadre du projet CIGALE; une œuvre en réalité virtuelle de Judith Guez qui testait la superposition d'un décor physique et d'un décor modélisé ; une composition électroacoustique développée au sein des EnsadLabs. Malgré la présence de quelques illustrations, il est difficile pour le lecteur d'en pénétrer l'esthétique immersive et interactive, mais surtout, rien ne lui donne le sentiment d'une continuité historique. Au lieu de souligner la présence souterraine de la stéréoscopie photographique ou cinématographique dans les dispositifs numériques que sont la VR et le son binaural, la juxtaposition des cas d'études n'engage qu'une réflexion, en touche impressionniste, à propos de l'illusion et de la virtualité. Cette dernière partie aurait grandement gagné à être remise en perspective par quelques remarques conclusives - critique qui vaut d'ailleurs pour l'ensemble de l'ouvrage car aucune conclusion générale ne vient le ressaisir dans sa totalité. La lecture de cette quatrième et dernière partie achève donc de nous rendre évident le fait suivant: le projet d'une "archéologie des médias " annoncée dans l'introduction de l'ouvrage Stétéoscopie et Illusion est un horizon théorique esquissé et à poursuivre. Face à un tel constat, libre à chacun de déplorer un retard de la pensée critique vis-à-vis des « arts trompeurs » contemporains ou bien d'y voir la possibilité même d'un émerveillement.

\section{AUTEURS}

\section{MARIE AUGER}

Université Paris 1-Panthéon-Sorbonne, HiCSA 